TITLE:

\title{
Amplitude dependence of frequency sweep rates of whistler mode chorus emissions
}

$\operatorname{AUTHOR}(S)$ :

Katoh, Yuto; Omura, Yoshiharu

\section{CITATION:}

Katoh, Yuto ... [et al]. Amplitude dependence of frequency sweep rates of whistler mode chorus emissions. JOURNAL OF GEOPHYSICAL RESEARCH-SPACE PHYSICS 2011, 116(A7): A07201.

ISSUE DATE:

2011-07

URL:

http://hdl.handle.net/2433/163428

RIGHT:

(C)2011. American Geophysical Union. 


\title{
Amplitude dependence of frequency sweep rates of whistler mode chorus emissions
}

\author{
Yuto Katoh ${ }^{1}$ and Yoshiharu Omura ${ }^{2}$ \\ Received 22 January 2011; revised 30 March 2011; accepted 5 April 2011; published 2 July 2011.
}

[1] The frequency and amplitude characteristics of chorus emissions are studied by performing electron hybrid code simulations with different initial number densities of energetic electrons. Chorus emissions with rising tones are generated in all simulation runs except for the simulation assuming the lowest number density. The frequency sweep rates of reproduced chorus vary depending on the variation of the wave amplitude of respective chorus elements. We find that the theoretically estimated frequency sweep rates are consistent with the simulation results. The simulation results reveal that the characteristic frequency variation of chorus elements showing rising tones are formed at the region very close to the magnetic equator while the wave amplitudes of chorus elements are significantly amplified through their propagation away from the equator. The spatial scale of the region where the observed explosive wave growth varies in each simulation run, corresponding to the difference of the wave amplitudes of reproduced chorus elements. We estimate the spatial scale $h_{c}$, a measure of the spatial extent of the generation region derived from the nonlinear wave growth theory, using parameters of the simulation runs and find its consistency with the simulation results. We also analyze the energy spectra of reproduced chorus elements in the simulation results and find that the spectra of chorus are essentially different from those predicted by the linear growth rates in the frequency domain. These results clearly demonstrate that the nonlinear wave-particle interaction governs the chorus generation mechanism.

Citation: Katoh, Y., and Y. Omura (2011), Amplitude dependence of frequency sweep rates of whistler mode chorus emissions, J. Geophys. Res., 116, A07201, doi:10.1029/2011JA016496.

\section{Introduction}

[2] Whistler mode chorus emissions are narrow band electromagnetic emissions observed mostly on the dawnside of the Earth's magnetosphere as a group of coherent wave elements often consisting of rising tones [Santolik et al., 2003, 2008]. A variety of chorus of falling tones and hooks has also been observed [Tsurutani and Smith, 1974; Chum et al., 2007, 2009]. Results of in situ observations reveal that chorus emissions are generated in the equatorial region of the inner magnetosphere and that the activity is enhanced during geomagnetically disturbed periods [Tsurutani and Smith, 1974; Meredith et al., 2001; Li et al., 2010]. In space weather research, the importance of chorus studies has been emphasized for a decade or more because of possible roles of chorus emissions in controlling the flux of relativistic electrons in the outer radiation belt during the recovery phase of geospace storms [Summers and Ma, 2000; Albert, 2002; Miyoshi et al., 2003; Horne et al., 2003; Summers

\footnotetext{
${ }^{1}$ Department of Geophysics, Graduate School of Science, Tohoku University, Sendai, Japan.

${ }^{2}$ Research Institute for Sustainable Humanosphere, Kyoto University, Uji, Japan.

Copyright 2011 by the American Geophysical Union. 0148-0227/11/2011JA016496
}

et al., 2007a, 2007b; Katoh and Omura, 2007b; Katoh et al., 2008; Kasahara et al., 2009].

[3] For the generation process of whistler mode waves in the inner magnetosphere, gyroresonant interactions have been studied between whistler mode waves and energetic electrons forming an anisotropic velocity distribution in a kinetic energy range from a few $\mathrm{keV}$ to tens of $\mathrm{keV}$ [Kennel and Petschek, 1966; Xiao et al., 1998]. Nonlinear effects in the presence of a coherent whistler mode wave have been investigated for explanation of the frequency variation of chorus elements [Nunn, 1974; Nunn et al., 1997; Trakhtengerts, 1995, 1999]. Nunn et al. [2005] discussed properties of triggered emissions and showed that the plasma density is an important factor determining the frequency sweep rates. Nunn et al. [2009] conducted numerical simulations of triggered emissions and reproduced triggering processes of rising and falling tones. Recently the generation process of whistler mode chorus emissions with rising tones has been reproduced by the electron hybrid simulation [Katoh and Omura, 2007a; Omura et al., 2008] and a fullparticle electromagnetic simulation [Hikishima et al., 2009]. The simulation results showed that chorus elements with rising tones are successively generated at the magnetic equator. The generated chorus elements propagating away from the equator undergo further nonlinear growth, while the 
Table 1. Summary of the Simulation Results ${ }^{\mathrm{a}}$

\begin{tabular}{lccccc}
\hline & Run 1 & Run 2 & Run 3 & Run 4 & Run 5 \\
\hline$N_{e, \text { hot }} / N_{e, \text { cold }}$ & $9.6 \times 10^{-4}$ & $8.8 \times 10^{-4}$ & $8.0 \times 10^{-4}$ & $7.2 \times 10^{-4}$ & $6.4 \times 10^{-4}$ \\
$\partial \omega / \partial t\left[\Omega_{e 0}^{2}\right]$ & $7.08 \times 10^{-5}$ & $5.72 \times 10^{-5}$ & $5.38 \times 10^{-5}$ & $3.72 \times 10^{-5}$ & - \\
Estimated $\partial \omega / \partial t\left[\Omega_{e 0}^{2}\right]$ & $9.35 \times 10^{-5}$ & $6.54 \times 10^{-5}$ & $5.39 \times 10^{-5}$ & $3.87 \times 10^{-5}$ & $2.95 \times 10^{-5}$ \\
\hline
\end{tabular}

${ }^{a}$ The ratio of the number density of energetic electrons to cold electrons at the magnetic equator, the averaged values of frequency sweep rates of chorus elements, and sweep rates estimated by equation (1).

wave frequency of part of the element is almost constant during its propagation. This result revealed that the frequency variation of chorus elements takes place at the region very close to the magnetic equator.

[4] Based on the analyses of the simulation results, the nonlinear wave growth theory has been proposed for the generation mechanism of whistler mode chorus emissions [Omura et al., 2008, 2009]. From the theoretical consideration of the dynamics of resonant electrons interacting with coherent whistler mode waves, it has been found that an electromagnetic electron hole is formed in the velocity phase space, resulting in a resonant current $J_{E}$ that causes wave growth of whistler mode waves. Omura et al. [2008] derived the relativistic inhomogeneity ratio describing the scale size of the electromagnetic electron hole in the velocity phase space, and they found that the magnitude of the resonant current $J_{E}$ maximizes at the specific inhomogeneity ratio. The expression of the relativistic inhomogeneity ratio consists of a term related to the spatial inhomogeneity of the background magnetic field and a term related to the frequency variation of whistler mode waves. Here we recall that the frequency variation of chorus elements in the simulation result only occurred at the magnetic equator where the spatial inhomogeneity of the background magnetic field is equal to zero. Based on the analysis of the simulation, Omura et al. [2008] proposed the nonlinear wave growth theory describing that the specific wave phase variation satisfying the condition of the maximum resonant currents undergoes significant amplification due to the effect of the selective nonlinear growth.

[5] The frequency sweep rates of chorus elements observed in the magnetosphere have been studied statistically in association with the plasma density [Macúšová et al., 2010] and wave amplitude [Cully et al., 2011]. The nonlinear wave growth theory shows that the frequency sweep rate of a chorus element depends on the amplitude of the coherent component of whistler mode waves generated through the instability driven by temperature anisotropy of energetic electrons near the magnetic equator. In the present study, we performed a series of simulations with different number densities of energetic electrons to check the validity of the nonlinear wave growth theory.

\section{Simulation Model}

[6] Omura et al. [2008] derived the relativistic secondorder resonance condition for a whistler mode wave with a varying frequency and showed that the frequency sweep rate is proportional to the wave amplitude near the magnetic equator. They found from the second-order resonance con- dition at the equator that the sweep rate of the wave frequency $\omega$ is described as

$$
\frac{\partial \omega}{\partial t}=\frac{0.4 \delta}{\gamma \xi} \frac{V_{\perp 0}}{c} \frac{\omega}{\Omega_{e}}\left(1-\frac{V_{R}}{V_{g}}\right)^{-2} \frac{B_{W}}{B_{0}} \Omega_{e}^{2},
$$

where $\delta^{2}=\left(1+\xi^{2}\right)^{-1}, c$ is the speed of light, $\gamma=\left[1-\left(v_{\|}^{2}+\right.\right.$ $\left.\left.v_{\perp}^{2}\right) / c^{2}\right]^{-1 / 2}, v_{\|}$and $v_{\perp}$ are parallel and perpendicular velocity components of an electron, $\xi^{2}=\omega\left(\Omega_{e}-\omega\right) / \omega_{p e}^{2}, \omega_{p e}$ is the electron plasma frequency, $V_{\perp 0}$ is an average value of $v_{\perp}$ of the initial velocity distribution of energetic electrons at the magnetic equator, the electron cyclotron frequency $\Omega_{e}=$ $e B_{0} / m_{0}, e$ and $m_{0}$ are the charge and the rest mass of an electron, $B_{0}$ is the background magnetic field, $V_{R}$ is the resonance velocity, $V_{g}$ is the group velocity, and $B_{W}$ is the amplitude of the wave magnetic field. Equation (1) tells us that the frequency sweep rate of chorus elements depends on the amplitude of the wave magnetic field near the magnetic equator.

[7] We use an electron hybrid code with a dipole magnetic field [Katoh and Omura, 2006, 2007a, 2007b; Omura et al., 2008]. We treat cold electrons as a fluid and energetic electrons as particles by the standard particle-in-cell (PIC) method. In this model we assume a spatially onedimensional system along the background magnetic field direction with a variable $h$ as a distance from the magnetic equator. Although the simulation system is one dimensional, the mirror motion of energetic electrons in the dipole magnetic field is taken into account by using a cylindrical field model [Katoh and Omura, 2006]. We solve Maxwell's equations with the current densities computed from the motion of the cold electron fluid and energetic electrons. We treat purely transverse electromagnetic waves so as to reproduce whistler mode waves propagating parallel to the dipole magnetic field, while we neglect the longitudinal electrostatic field.

[8] The time step and the grid spacing of the simulation are $0.01 \Omega_{e 0}^{-1}$ and $0.06 c \Omega_{e 0}^{-1}$, respectively, where $\Omega_{e 0}$ is the electron gyrofrequency at the magnetic equator. The simulation system is along the field line with $L_{h}=16384$ grid points. Number of particles initially placed in the simulation system representing energetic electrons is $4096 \times L_{h}$. We assume the plasma frequency of the cold electrons $\omega_{p e}$ at the equator is $4 \Omega_{e 0}$, while the number density of cold electrons $N_{e, \text { cold }}$ is constant in space and time.

[9] We assume that the initial velocity distribution $f\left(v_{\|}, v_{\perp}\right)$ of energetic electrons at the magnetic equator forms an anisotropic velocity distribution with a loss cone, given by

$$
f\left(v_{\|}, v_{\perp}\right)=C \exp \left(-\frac{v_{\|}^{2}}{2 V_{t \|}^{2}}\right) g\left(v_{\perp}\right)
$$




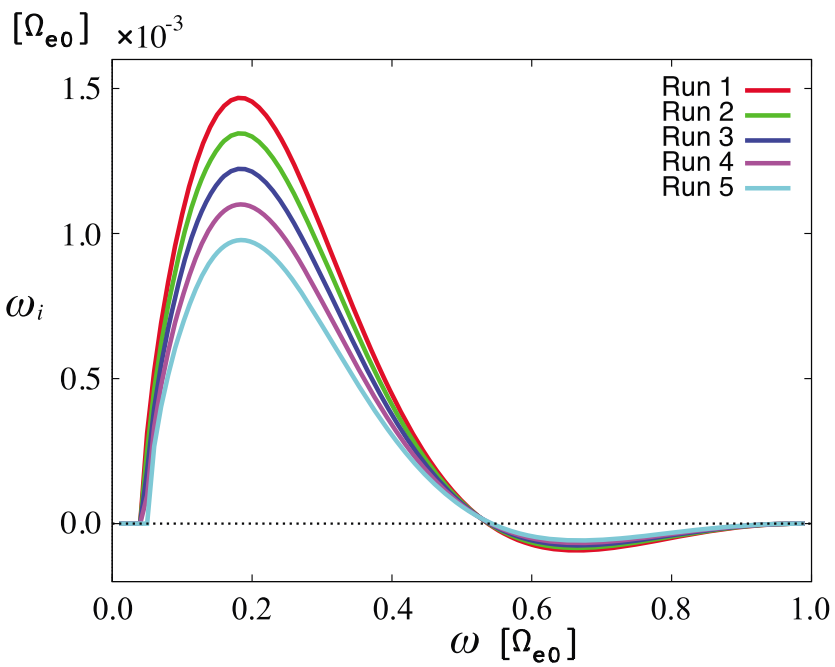

Figure 1. The linear growth rates $\omega_{i}$ of whistler mode waves as a function of wave frequency calculated from the initial settings of the simulations with the number densities of energetic electrons as shown in Table 1.

and

$$
g\left(v_{\perp}\right)=\frac{1}{1-\beta}\left\{\exp \left(-\frac{v_{\perp}^{2}}{2 V_{t \perp}^{2}}\right)-\exp \left(-\frac{v_{\perp}^{2}}{2 \beta V_{t \perp}^{2}}\right)\right\},
$$

where $C$ is a constant, $\beta=0.5, V_{t \|}$ and $V_{t \perp}$ are thermal velocities of energetic electrons in the direction parallel and perpendicular to the background magnetic field. We assume the thermal velocities $V_{t \|}$ and $V_{t \perp}$ are $0.225 c$ and $0.6 c$. The temperature anisotropy $T_{\perp} / T_{\|}\left(=V_{t \perp}^{2} / V_{t \|}^{2}\right)$ assumed for the velocity distribution of energetic electrons drives an instability generating a band of whistler mode waves. As has been studied by Hikishima et al. [2009], the linear growth rate of the whistler mode instability is determined by the temperature anisotropy and is proportional to the ratio of the number density of energetic electrons $N_{e, \text { hot }}$ to cold electrons $N_{e \text {,cold. }}$. In the present study, we perform five simulation runs with different $N_{e \text {,hot }} / N_{e \text {,cold }}$ under the same $T_{\perp} / T_{\|}$to investigate the characteristics of chorus emissions emerging from whistler mode waves of different wave amplitudes through the different linear growth phases. The density ratio assumed in each run is given in Table 1, while the parameters used in run 3 is the same as those of the previous study [Katoh and Omura, 2007a, 2007b]. Figure 1 shows the linear growth rates $\omega_{i}$ of whistler mode waves given by the relativistic linear theory of Xiao et al. [1998] from the initial settings of simulations as described above and number densities of energetic electrons as given in Table 1. The estimated max- imum linear growth rates take the maximum values at the frequency of $0.18 \Omega_{e 0}$, which are listed in Table 2 . They vary depending on the number densities of energetic electrons assumed in the simulations.

\section{Results and Discussion}

[10] Figure 2 shows the frequency spectra of the wave electric field observed in the simulation results. A band of whistler mode waves is generated in the early stage of the simulation through an instability driven by the temperature anisotropy, and the amplitudes of these incoherent whistler mode waves grow with the timescale corresponding to the linear growth rates. We find that chorus elements with rising tones are generated in all simulation runs except for run 5 and that there are distinct differences in the frequency sweep rates of chorus elements among the simulations. As shown in Figure 2, whistler mode waves enhanced in the simulations are classified into two components according to the spectral characteristics; coherent chorus elements and a band of incoherent waves. While these two components are overlapped in the lower frequency range of Figure 2, coherent chorus elements dominate in the frequency range higher than $0.23 \Omega_{e 0}$, corresponding to the wave number range $k>2.2 c^{-1} \Omega_{e 0}$. Figure 3 shows the evolutions of the wave magnetic field amplitude in the simulation system, while the long-wavelength modes $\left(k<2.2 c^{-1} \Omega_{e 0}\right)$ have been eliminated in order to extract chorus elements. Dashed lines of Figure 4 show time histories of wave magnetic field amplitude $B_{W}$ averaged over $h=-100 \sim 100 c \Omega_{e 0}^{-1}$, and solid lines represent the averaged wave amplitudes of coherent chorus elements. In the early stage of the simulation, the evolution of the whistler mode instability is indicated by the exponential growth of the averaged wave amplitude with the linear growth rate. The wave amplitude saturates at a certain level at $t=2500 \Omega_{e 0}^{-1}$, and then the tendency of the amplitude variation changes to the abrupt oscillation showing the signature of the nonlinear growth. Therefore we assume the termination of the linear growth phase is $t=2500 \Omega_{e 0}^{-1}$. After the saturation of the linear growth phase, the nonlinear growth of coherent chorus elements emerged. As shown by time histories of the wave magnetic field amplitude in Figure 4, the saturation levels of the linear growth are different among the simulation runs. Although the difference of the saturation levels would be explained by the difference of the initial number densities of energetic electrons, precise comparison is difficult because of the nonlinear amplification simultaneously occurs in the time interval of the linear growth saturation.

[11] The simulation results reveal that the characteristic frequency variation of chorus elements showing rising tones has been formed at the region very close to the magnetic

Table 2. Summary of the Maximum Values of the Estimated Linear Growth Rates $\omega_{i}$, Nonlinear Growth Rates $\Gamma_{N}$ Under the Initial Conditions of Simulation Runs and $\boldsymbol{h}_{\boldsymbol{c}}$ Estimated from the Simulation Results ${ }^{\mathrm{a}}$

\begin{tabular}{lccccc}
\hline & Run 1 & Run 2 & Run 3 & Run 4 & Run 5 \\
\hline$\omega_{i}\left[\Omega_{e 0}\right]$ & $1.47 \times 10^{-3}$ & $1.35 \times 10^{-3}$ & $1.22 \times 10^{-3}$ & $1.10 \times 10^{-3}$ & $9.77 \times 10^{-4}$ \\
$\Gamma_{N}\left[\Omega_{e 0}\right]$ & $3.37 \times 10^{-3}$ & $2.83 \times 10^{-3}$ & $2.34 \times 10^{-3}$ & $1.89 \times 10^{-3}$ & $1.50 \times 10^{-3}$ \\
$h_{c}\left[\mathrm{c} / \Omega_{e 0}\right]$ & 209 & 139 & 105 & 83 & 68 \\
\hline
\end{tabular}

${ }^{a}$ These maximum growth rates are found at $0.18 \Omega_{e 0}$ for $\omega_{i}$ and at $0.32 \Omega_{e 0}$ for $\Gamma_{N}$. The values of $h_{c}$ are averaged over the time interval from 4000 to $5000 \Omega_{e 0}^{-1}$ of the simulation runs. 
(a)

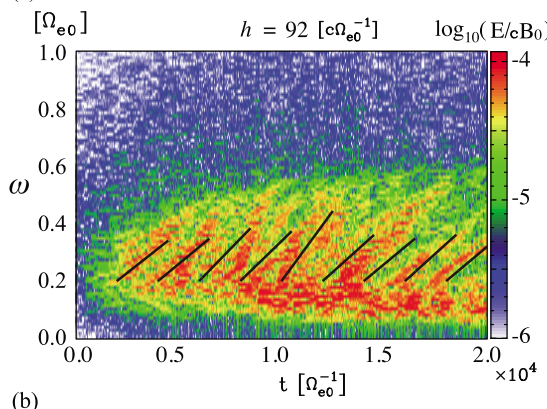

(b)

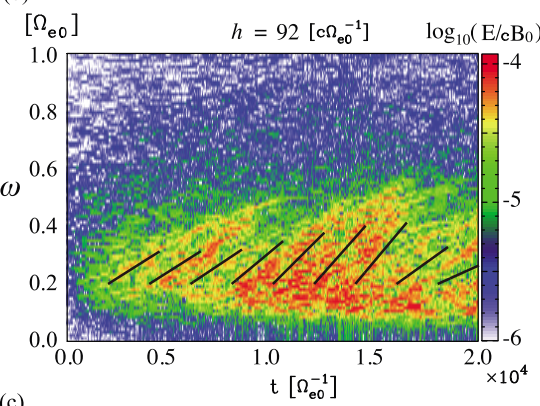

(c)

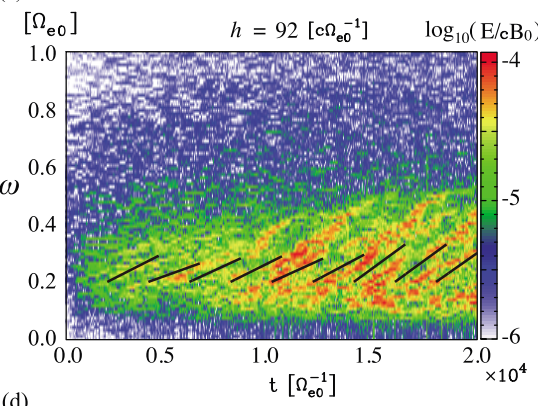

(d)

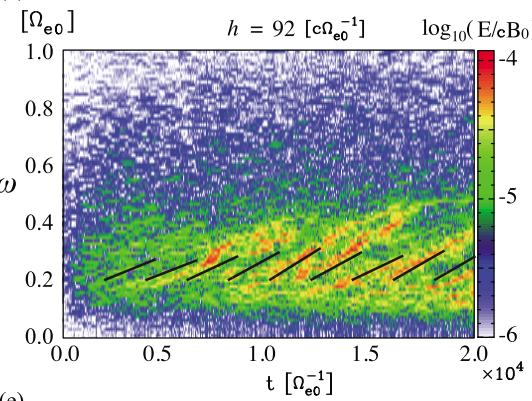

(e)

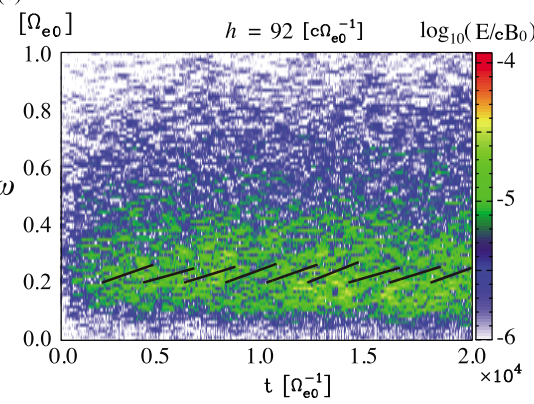

Figure 2. Dynamic spectra of the wave electric field $\left(E_{\perp}\right)$ observed in the Northern Hemisphere $\left(h=+92 c \Omega_{e 0}^{-1}\right)$ in (a) run 1, (b) run 2, (c) run 3, (d) run 4, and (e) run 5. Frequency sweep rates estimated theoretically from an instantaneous wave magnetic field amplitude of each interval are also shown by solid lines.
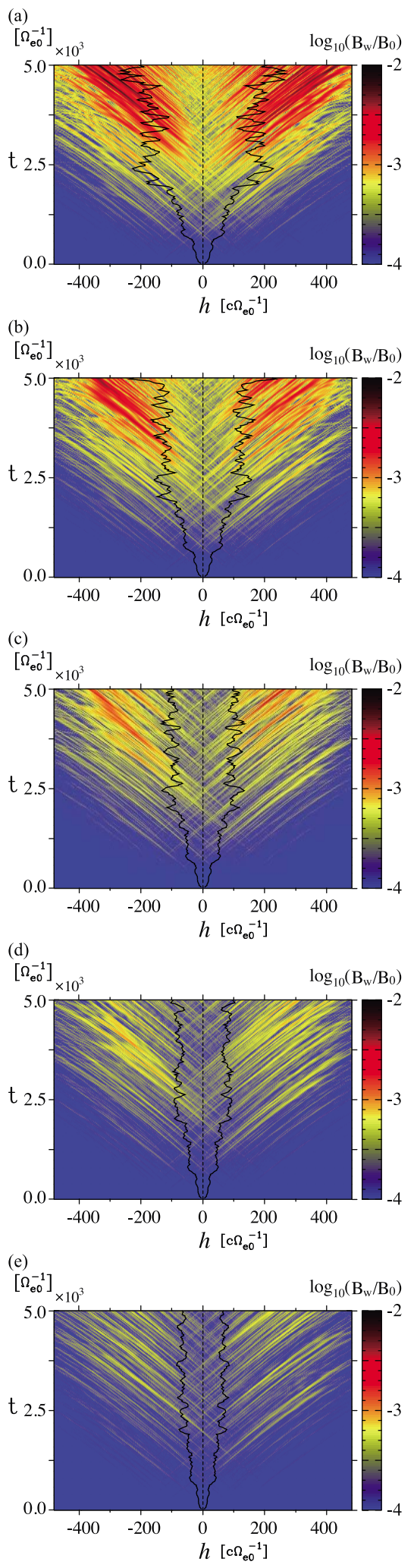

Figure 3. Wave amplitude $B_{W}(h, t)$ of chorus emissions observed in (a) run 1 , (b) run 2 , (c) run 3 , (d) run 4 , and (e) run 5. The long-wavelength modes $\left(k<2.2 c^{-1} \Omega_{e 0}\right)$ have been eliminated. Solid lines in each panel show the location of the distance $\pm h_{c}(t)$ from the equator, while $h_{c}(t)$ is estimated from the wave magnetic field amplitude $B_{W}(h, t)$ averaged over $h=-10 \sim 10 c \Omega_{e 0}^{-1}$ in each run. 
(a) $\times 10^{-3}$

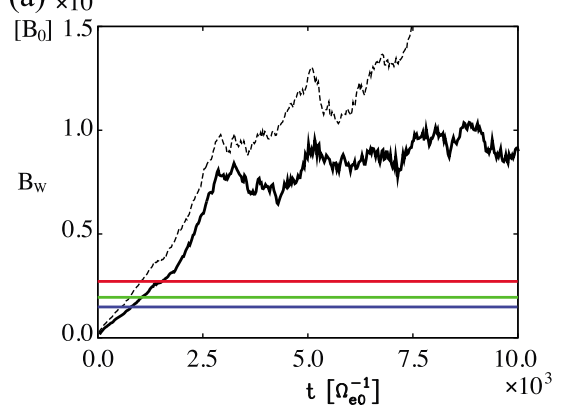

(b) $\times 10^{-3}$

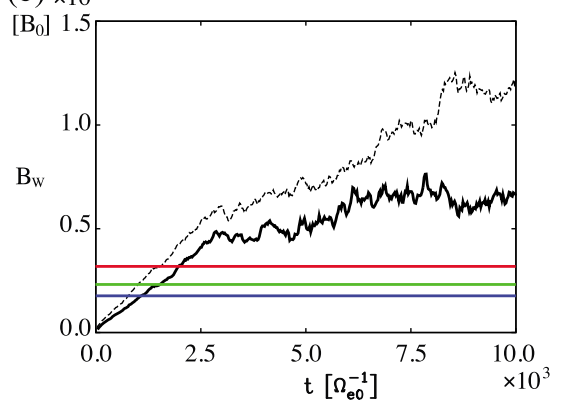

(c) $\times 10^{-3}$

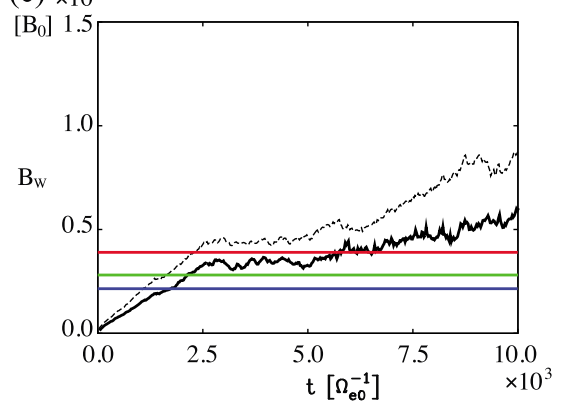

(d) $\times 10^{-3}$

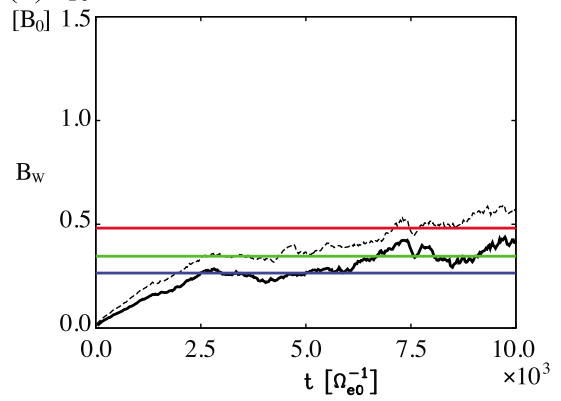

(e) $\times 10^{-3}$

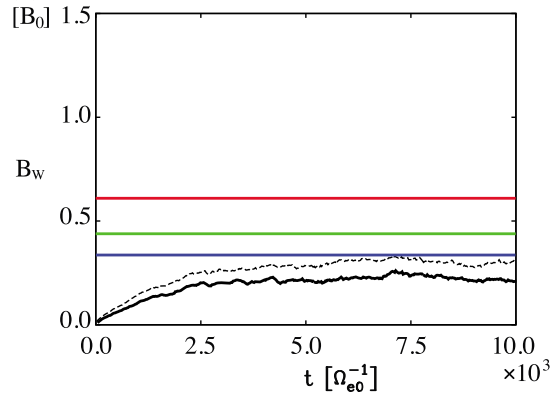

equator (within $10 c \Omega_{e 0}^{-1}$ ) while the wave amplitudes of elements have been significantly intensified through their propagation away from the equator $\left(\sim 100 c \Omega_{e 0}^{-1}\right)$. The spatial scale of the region where the explosive wave growth has been observed varies in each simulation run, corresponding different wave amplitudes of reproduced chorus elements. A measure of the spatial extent of the generation region has been derived by Omura et al. [2009] from the nonlinear wave growth theory. The inhomogeneity ratio $S$ is given by

$$
S=-\frac{1}{s_{0} \omega \Omega_{w}}\left(s_{1} \frac{\partial \omega}{\partial t}+c s_{2} \frac{\partial \Omega_{e}}{\partial h}\right),
$$

where

$$
\begin{gathered}
s_{0}=\frac{\delta}{\xi} \frac{V_{\perp 0}}{c}, \\
s_{1}=\gamma\left(1-\frac{V_{R}}{V_{g}}\right)^{2}, \\
s_{2}=\frac{1}{2 \xi \delta}\left\{\frac{\gamma \omega}{\Omega_{e}}\left(\frac{V_{\perp 0}}{c}\right)^{2}-\left[2+\Lambda \frac{\delta^{2}\left(\Omega_{e}-\gamma \omega\right)}{\Omega_{e}-\omega}\right] \frac{V_{R} V_{p}}{c^{2}}\right\},
\end{gathered}
$$

$\Omega_{w}=e B_{w} / m_{0}, \Lambda=1$ in the present study, and $V_{p}$ is the phase velocity of the whistler mode waves. The critical distance $h_{c}$ from the equator is defined as the location where the first and second term of the right-hand side of (4) become equal. By assuming a parabolic variation of the background magnetic field as expressed by $\Omega_{e}=\Omega_{e 0}\left(1+a h^{2}\right)$ near the magnetic equator, Omura et al. [2009] derived $h_{c}$ as

$$
h_{c}=\frac{s_{0} \omega \Omega_{w 0}}{5 \operatorname{cas}_{2} \Omega_{e 0}}
$$

where $\Omega_{w 0}=e B_{w 0} / m_{0}, B_{w 0}$ is the wave magnetic field amplitude normalized by $B_{0}$ at the equator, and $a=9.8 \times 10^{-7}$ $\left(c^{-1} \Omega_{e 0}\right)^{2}$ in the simulation system assumed in the present study. Using (8), we examined the spatial scale $h_{c}$ from parameters of each simulation run. In estimating $h_{c}$, we used time histories of the wave magnetic field amplitudes averaged over $h=-10 \sim 10 c \Omega_{e 0}^{-1}$ of Figure 3 as $B_{w 0}$, which correspond to the wave amplitudes of coherent chorus elements appeared in the region close to the magnetic equator in the simulation results. We also use the wave frequency 0.32 $\Omega_{e 0}$ corresponding to the frequency of the maximum nonlinear growth rate which will be discussed later. We show the distance $\pm h_{c}(t)$ from the equator in Figures $3 \mathrm{a}-3 \mathrm{e}$ and values of $h_{c}$ averaged over the time interval from 4000 to $5000 \Omega_{e 0}^{-1}$ in Table 2. The estimated $h_{c}$ in each run is consistent with the

Figure 4. Wave magnetic field amplitude observed in (a) run 1 , (b) run 2 , (c) run 3 , (d) run 4 , and (e) run 5. Dashed lines show time histories of wave magnetic field amplitude $B_{W}$ averaged over $h=-100 \sim 100 c \Omega_{e 0}^{-1}$ in each run. Solid lines represent the averaged wave amplitude after eliminating the long-wavelength modes $\left(k<2.2 c^{-1} \Omega_{e 0}\right)$. Estimated threshold values of the wave magnetic field amplitude are also shown by horizontal red $\left(\omega=0.18 \Omega_{e 0}\right)$, green $\left(0.2 \Omega_{e 0}\right)$, and blue lines $\left(0.22 \Omega_{e 0}\right)$ for the case of $Q=0.5$. 


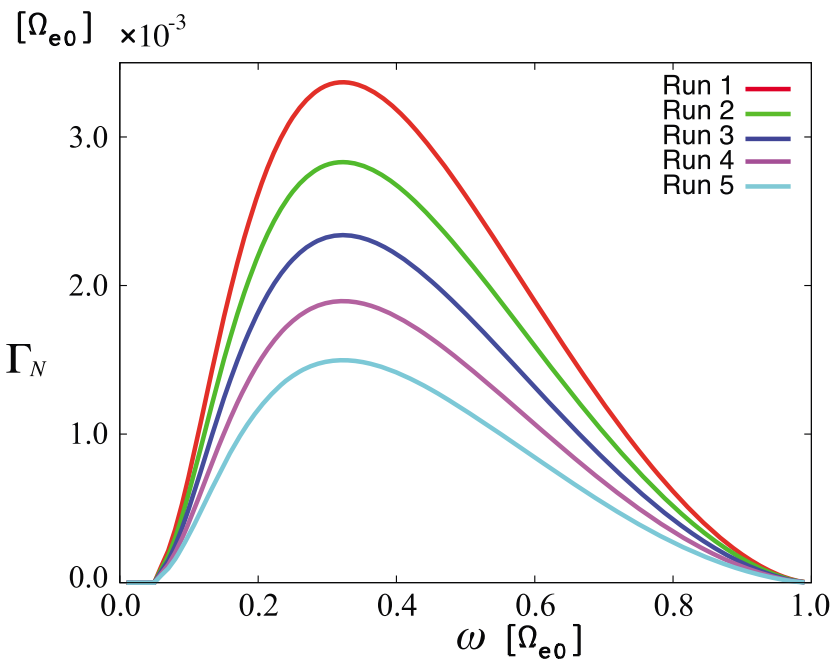

Figure 5. The nonlinear growth rates $\Gamma_{N}$ as a function of wave frequency. The initial settings of the simulations and the estimated threshold values $B_{w, t h}$ for the case of $Q=$ 0.5 and $\omega=0.18 \Omega_{e 0}$ are used.

spatial extent of the region where we observe the nonlinear wave growth due to the frequency sweep rate of chorus elements. Beyond the distance $h_{c}$ from the equator, the nonlinear wave growth is maintained by the spatial gradient of the magnetic field.

[12] As shown in Figure 2 of Omura et al. [2008], the magnitude of the resonant current $J_{E}$ maximizes at $S=-0.4$, while at the magnetic equator the term related to the frequency sweep rate $\partial \omega / \partial t$ only contributes to $S$. The formation of $J_{E}$ with the maximum magnitude results in the explosive growth of coherent waves having a specific phase variation $\partial \omega / \partial t$ satisfying the condition $S=-0.4$. Based on the nonlinear wave growth theory, the condition described above is maintained during the chorus generation process in the simulation results, and a larger wave amplitude results in a faster frequency variation. Here we estimate the frequency sweep rate by (1) showing the relationship between the frequency sweep rate and the wave magnetic field amplitude. In evaluating the frequency sweep rate from (1), we note that we assume the presence of a coherent wave in deriving a series of equations of the nonlinear wave growth theory. Therefore, since the frequency sweep rate of chorus elements should be examined by the wave amplitude of coherent components observed in the region close to the magnetic equator, time histories of the wave magnetic field amplitude averaged over $h=-10 \sim 10 c \Omega_{e 0}^{-1}$ of Figure 3 are used to estimate the theoretical frequency sweep rate by (1). We evaluated frequency sweep rates at a certain time interval of $2000 \Omega_{e 0}^{-1}$ in the simulations and superposed them on the spectra in Figure 2. We find the consistency of (1) by comparing the spectral characteristics of the reproduced chorus and the estimated sweep rates. The averaged values of the frequency sweep rates of the reproduced chorus elements and theoretical estimations are summarized in Table 1.

[13] As shown in Figure 2e, distinct chorus elements are not observed in run 5 revealing that there is a certain threshold of the wave amplitude in the chorus generation process. The threshold of the wave amplitude for the nonlinear wave growth of chorus emissions has been theoretically derived by Omura et al. [2009], and the equation of the threshold $B_{w, t h}$ is given by

$$
\frac{B_{w, t h}}{B_{0}}=\tilde{\Omega}_{t h}
$$

where

$$
\tilde{\Omega}_{t h}=\frac{100 \pi^{3} \gamma^{3} \xi}{\tilde{\omega} \tilde{\omega}_{p h}^{4} \tilde{V}_{\perp 0}^{5} \delta^{5}}\left(\frac{\tilde{a} s_{2} \tilde{U}_{t \|}}{Q}\right)^{2} \exp \left(\frac{\gamma^{2} \tilde{V}_{R}^{2}}{\tilde{U}_{t \|}^{2}}\right),
$$

$\tilde{\omega}=\omega / \Omega_{e 0}, \tilde{\omega}_{p h}=\omega_{p h} / \Omega_{e 0}, \omega_{p h}=\omega_{p e}\left(N_{e, h o t} / N_{e, \text { cold }}\right)^{1 / 2}, \tilde{V}_{\perp 0}=$ $V_{\perp 0} / c, \tilde{a}=a c^{2} / \Omega_{e 0}^{2}, \tilde{U}_{t \|}=U_{t \mid} / c=\gamma V_{t||} / c$, and $Q$ represents the depth of the electromagnetic electron hole in the velocity phase space. Equation (10) is derived from the consideration of the optimum self-sustaining nonlinear wave growth satisfying the condition of $S=-0.4$ through propagation of chorus elements with rising tones along the dipole magnetic field. Considering the formation process of the nonlinear resonant current $J_{E}$, the amplitude of $J_{E}$ is determined by both the size of the trapping region and the population of resonant electrons in the trapping region. Since the resonant currents are formed by the modulation of the distribution of resonant electrons in the velocity phase space due to the presence of the electron hole, a large size of the trapping region and less population of trapped electrons result in the strong resonant current formation. Horizontal lines in red, green, and blue in Figure 4 show the threshold values $B_{w, t h}$ estimated from (10) with $Q=0.5$ at different frequencies $\omega=0.18,0.2$, and $0.22 \Omega_{e 0}$, respectively. As the frequency increases, $B_{w, t h}$ decreases rapidly. The nonlinear wave growth occurs after the amplitude of a coherent component of waves exceeds $B_{w, t h}$. We consider that coherent components of waves emerge from a band of whistler mode waves after the saturation of the linear growth and that the starting frequency of a rising tone chorus element is determined by the frequency at which the wave amplitude exceeds $B_{w, t h}$. Therefore, it is possible that the nonlinear wave growth starts from a frequency slightly higher than the frequency of the maximum linear growth rate shown in Figure 1. By comparing the wave amplitude and estimated threshold shown in Figure 4, we conclude that (10) can give the threshold of the wave amplitude for generating chorus emissions with rising tones.

[14] From consideration of the condition to corresponding the maximum resonant current $J_{E}$ and resultant gain of wave magnetic field, Omura et al. [2009] defined the nonlinear growth rate $\Gamma_{N}$ as

$$
\Gamma_{N}=\frac{Q \omega_{p h}^{2}}{2}\left(\frac{\xi}{\Omega_{w} \omega}\right)^{1 / 2} \frac{V_{g}}{U_{t \|}}\left(\frac{V_{\perp 0} \delta}{c \pi \gamma}\right)^{3 / 2} \exp \left(-\frac{\gamma^{2} V_{R}^{2}}{2 U_{t \|}^{2}}\right) .
$$

We evaluated $\Gamma_{N}$ by referring to the initial settings of the simulations and the estimated threshold values $B_{w, t h}$ as shown in Figure 4. Figure 5 shows the estimated $\Gamma_{N}$ for each simulation as a function of wave frequency. Table 2 gives maximum values of $\Gamma_{N}$ found at the frequency of $0.32 \Omega_{e 0}$. By comparing Figures 1 and 5 and the maximum values of growth rates shown in Table 2, we find distinct differences 


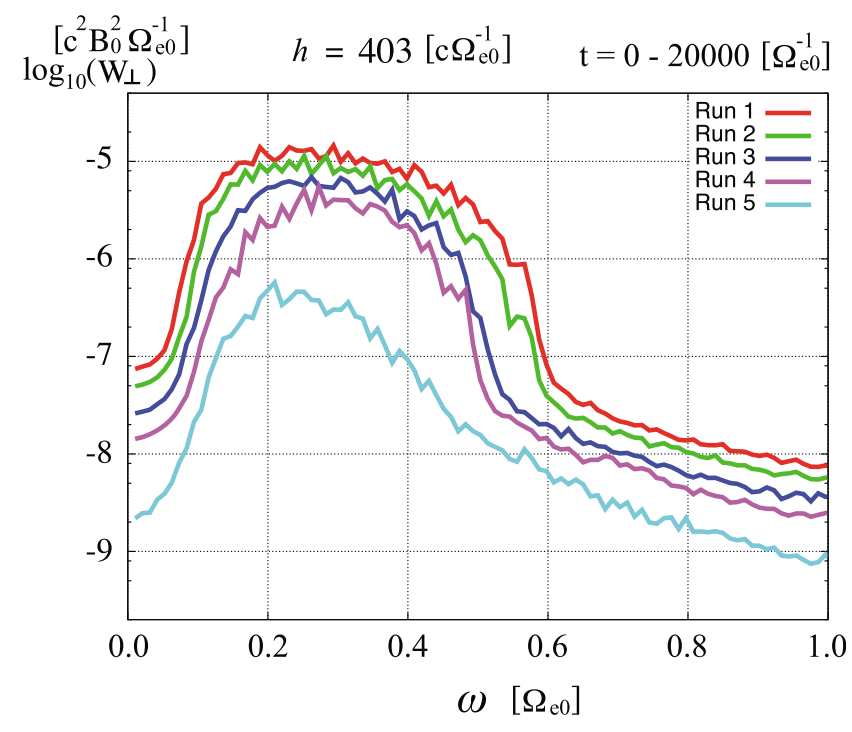

Figure 6. Frequency spectra $W_{\perp}(\omega)$ of the wave electric field power $E_{\perp}^{2}(\omega)$ integrated over the time interval $t=0 \sim$ $20000 \Omega_{e 0}^{-1}$ observed at $h=+403 c \Omega_{e 0}^{-1}$.

between the predictions obtained from the linear theory and nonlinear wave growth theory. The maximum values of $\Gamma_{N}$ are significantly higher than those of $\omega_{i}$. Besides the frequency profiles of $\Gamma_{N}$ reveal that explosive wave amplification occurs in the wider frequency range than those predicted by the linear theory. We find that these estimations are consistent with the simulation results. However, the nonlinear growth rates are positive up to the electron cyclotron frequency, while the rising tone emissions in the simulation results are terminated around $0.6 \Omega_{e 0}$. These results reveal that there is a saturation mechanism of the nonlinear wave growth, which is left as a future study. We also find that we obtained positive values of $\Gamma_{N}$ for the case of run 5 in which we did not observe distinct chorus elements. Considering that we assume the presence of a coherent wave in the nonlinear wave growth theory, we can explain this discrepancy by referring to Figure 4e that the amplitude of whistler mode waves generated in the linear stage of run 5 was smaller than the threshold level required for emergence of the nonlinear wave growth. The threshold level of the nonlinear wave growth has also been discussed in detail by Omura and Nunn [2011].

[15] Figure 6 shows the energy spectra $W_{\perp}(\omega)$ of the wave electric field power $E_{\perp}^{2}(\omega)$ integrated over the time interval $t=0 \sim 20000 \Omega_{e 0}^{-1}$ observed at $h=+403 c \Omega_{e 0}^{-1}$, clarifying the difference of wave power of chorus emissions reproduced in the simulation results. Especially by comparing the result of run 5 (no distinct chorus element) with other runs, we find that the emergence of the nonlinear wave growth in the chorus generation process results in the sharp increase of the wave power in the frequency range of chorus elements. Figure 6 clearly demonstrates that the nonlinear waveparticle interaction governs the chorus generation mechanism and that the frequency spectra of wave power in the chorus emissions are significantly different from those expected by the linear theory.

[16] In evaluating (10) and (11), we assumed $Q=0.5$. The quantitative evaluation of $Q$ from the simulation result is important but slightly difficult. The electromagnetic electron hole is formed in the velocity phase space $\left(v_{\|}-\zeta\right.$ space $)$ centered at $V_{R}$. Since high energy electrons with different values of the Lorentz factor give different values of $V_{R}$, the trapping regions overlap if we draw the distribution in the $v_{\|}-\zeta$ space using the simulation results. The overlap of the trapping regions in the different energy range makes it difficult to obtain the quantity of $Q$ from the distribution of energetic electrons in the velocity phase space in the simulation result.

[17] In the present study, reproduced chorus emissions consist of rising tones. Observation results revealed some of chorus elements show falling tones [Tsurutani and Smith, 1974; Chum et al., 2007, 2009], and previous studies suggested that the nonlinear wave-particle interactions play important roles in the generation process of falling tones as well [Nunn et al., 2009]. In addition, we treated waves propagating purely parallel to the background magnetic field. Although this assumption is basically reasonable in the chorus generation region, chorus emissions propagating obliquely from the source region have been observed [Santolik et al., 2009; Chum et al., 2009]. Studies based on the nonlinear wave growth theory for the generation mechanism of emissions with falling tones and effects of the oblique propagation are important and are left as future studies.

\section{Summary}

[18] We studied the frequency and amplitude characteristics of chorus emissions by performing the electron hybrid code simulations with different initial number densities of energetic electrons. We found in the simulation results that chorus emissions with rising tones are clearly generated in all simulation runs except for the simulation assuming the lowest number density. In the simulation results, the increase of the wave amplitude during the linear growth phase varied proportional to the assumed number density of energetic electrons. We obtained the wave amplitude of coherent waves by eliminating incoherent long-wavelength modes due to the linear instability, and we estimated the frequency sweep rate using the theoretical expression (1). The estimated sweep rates are consistent with those of chorus elements observed in the simulation results. We also studied the amplitude threshold for chorus emissions by comparing the simulation results with the estimation by (10), and we found that the values of the estimated threshold are consistent with the simulation results. The results of the present simulations demonstrate the validity of the nonlinear wave growth theory [Omura et al., 2008, 2009]. In addition we analyzed the frequency spectra of the chorus elements reproduced in the simulation results and found that the spectra of chorus emissions are essentially different from those predicted by the linear growth rates. The spectra of chorus elements are extended to the higher frequency range, in which the linear growth rates are negative. Although the nonlinear growth rates are positive up to the electron cyclotron frequency, the rising tone emissions are terminated around $0.6 \Omega_{e 0}$. The saturation mechanism of the nonlinear wave growth is left as a future study. 
[19] Acknowledgments. The computation was performed with supercomputers at Research Institute for Sustainable Humanosphere and Academic Center for Computing and Media Studies of Kyoto University and the Solar-Terrestrial Environment Laboratory of Nagoya University. This work was supported by Grants-in-Aid 20340135 and 22684025 and the Global COE program "Global Education and Research Center for Earth and Planetary Dynamics" at Tohoku University of the Ministry of Education, Science, Sports and Culture of Japan.

[20] Robert Lysak thanks the reviewers for their assistance in evaluating this paper.

\section{References}

Albert, J. M. (2002), Nonlinear interaction of outer zone electrons with VLF waves, Geophys. Res. Lett., 29(8), 1275, doi:10.1029/ 2001 GL013941.

Chum, J., O. Santolík, A. W. Breneman, C. A. Kletzing, D. A. Gurnett, and J. S. Pickett (2007), Chorus source properties that produce time shifts and frequency range differences observed on different Cluster spacecraft, J. Geophys. Res., 112, A06206, doi:10.1029/2006JA012061.

Chum, J., O. Santolík, D. A. Gurnett, and J. S. Pickett (2009), Oblique lower band chorus waves: Time shifts between discrete elements observed by the Cluster spacecraft, J. Geophys. Res., 114, A00F02, doi:10.1029/2009JA014366.

Cully, C. M., V. Angelopoulos, U. Auster, J. Bonnell, and O. Le Contel (2011), Observational evidence of the generation mechanism for risingtone chorus, Geophys. Res. Lett., 38, L01106, doi:10.1029/ 2010GL045793.

Hikishima, M., S. Yagitani, Y. Omura, and I. Nagano (2009), Full particle simulation of whistler-mode rising chorus emissions in the magnetosphere, J. Geophys. Res., 114, A01203, doi:10.1029/2008JA013625.

Horne, R. B., N. P. Meredith, R. M. Thorne, D. Heynderickx, R. H. A. Iles, and R. R. Anderson (2003), Evolution of energetic electron pitch angle distributions during storm time electron acceleration to megaelectronvolt energies, J. Geophys. Res., 108(A1), 1016, doi:10.1029/2001JA009165.

Kasahara, Y., Y. Miyoshi, Y. Omura, O. P. Verkhoglyadova, I. Nagano, I. Kimura, and B. T. Tsurutani (2009), Simultaneous satellite observations of VLF chorus, hot and relativistic electrons in a magnetic storm "recovery" phase, Geophys. Res. Lett., 36, L01106, doi:10.1029/ 2008GL036454

Katoh, Y. and Y. Omura (2006), A study of generation mechanism of VLF triggered emission by self-consistent particle code, J. Geophys. Res., 111, A12207, doi:10.1029/2006JA011704.

Katoh, Y. and Y. Omura (2007a), Computer simulation of chorus wave generation in the Earth's inner magnetosphere, Geophys. Res. Lett., 34, L03102, doi:10.1029/2006GL028594.

Katoh, Y. and Y. Omura (2007b), Relativistic particle acceleration in the process of whistler-mode chorus wave generation, Geophys. Res. Lett. 34, L13102, doi:10.1029/2007GL029758.

Katoh, Y., Y. Omura, and D. Summers (2008), Rapid energization of radiation belt electrons by nonlinear wave trapping, Ann. Geophys., 26, 3451-3456.

Kennel, C. F., and H. E. Petschek (1966), Limit on stable trapped particle fluxes, J. Geophys. Res., 71, 1-28, doi:10.1029/JZ071i001p00001.

$\mathrm{Li}$, W., et al. (2010), THEMIS analysis of observed equatorial electron distributions responsible for the chorus excitation, J. Geophys. Res., 115, A00F11, doi:10.1029/2009JA014845.

Macúšová, E., et al. (2010), Observations of the relationship between frequency sweep rates of chorus wave packets and plasma density, J. Geophys. Res., 115, A12257, doi:10.1029/2010JA015468.

Meredith, N. P., R. B. Horne, and R. R. Anderson (2001), Substorm dependence of chorus amplitudes: Implications for the acceleration of electrons to relativistic energies, J. Geophys. Res., 106, 13,165-13,178, doi:10.1029/2000JA900156.

Miyoshi, Y., A. Morioka, T. Obara, H. Misawa, T. Nagai, and Y. Kasahara (2003), Rebuilding process of the outer radiation belt during the 3 November
1993 magnetic storm: NOAA and EXOS-D observations, J. Geophys. Res., 108(A1), 1004, doi:10.1029/2001JA007542.

Nunn, D. (1974), A self-consistent theory of triggered VLF emissions, Planet. Space Sci., 22, 349-378, doi:10.1016/0032-0633(74)90070-1.

Nunn, D., Y. Omura, H. Matsumoto, I. Nagano, and S. Yagitani (1997), The numerical simulation of VLF chorus and discrete emissions observed on the Geotail satellite using a Vlasov code, J. Geophys. Res., 102(A12), 27,083-27,097, doi:10.1029/97JA02518.

Nunn, D., M. Rycroft, and V. Trakhtengerts (2005), A parametric study of the numerical simulations of triggered VLF emissions, Ann. Geophys. $23,3655-3666$

Nunn, D., O. Santolík, M. Rycroft, and V. Trakhtengerts (2009), On the numerical modelling of VLF chorus dynamical spectra, Ann. Geophys. $27,2341-2359$.

Omura, Y., and D. Nunn (2011), Triggering process of whistler mode chorus emissions in the magnetosphere, J. Geophys. Res., 116, A05205, doi:10.1029/2010JA016280.

Omura, Y., Y. Katoh, and D. Summers (2008), Theory and simulation of the generation of whistler-mode chorus, J. Geophys. Res., 113, A04223, doi:10.1029/2007JA012622

Omura, Y., M. Hikishima, Y. Katoh, D. Summers, and S. Yagitani (2009), Nonlinear mechanisms of lower-band and upper-band VLF chorus emissions in the inner magnetosphere, J. Geophys. Res., 114, A07217, doi:10.1029/2009JA014206.

Santolík, O., D. A. Gurnett, J. S. Pickett, M. Parrot, and N. CornilleauWehrlin (2003), Spatio-temporal structure of storm-time chorus, J. Geophys. Res., 108(A7), 1278, doi:10.1029/2002JA009791.

Santolík, O., E. Macusova, E. E. Titova, B. V. Kozelov, D. A. Gurnett, J. S. Pickett, V. Y. Trakhtengerts, and A. G. Demekhov (2008), Frequencies of wave packets of whistler-mode chorus inside its source region: A case study, Ann. Geophys., 26, 1665-1670.

Santolík, O., D. A. Gurnett, J. S. Pickett, J. Chum, and N. CornilleauWehrlin (2009), Oblique propagation of whistler mode waves in the chorus source region, J. Geophys. Res., 114, A00F03, doi:10.1029/ 2009JA014586.

Summers, D., and C. Ma (2000), A model for generating relativistic electrons in the Earth's inner magnetosphere based on gyroresonant waveparticle interactions, J. Geophys. Res., 105(A2), 2625-2639, doi:10.1029/1999JA900444.

Summers, D., B. Ni, and N. P. Meredith (2007a), Timescales for radiation belt electron acceleration and loss due to resonant wave-particle interactions: 1. Theory, J. Geophys. Res., 112, A04206, doi:10.1029/ 2006JA011801.

Summers, D., B. Ni, and N. P. Meredith (2007b), Timescales for radiation belt electron acceleration and loss due to resonant wave-particle interactions: 2. Evaluation for VLF chorus, ELF hiss, and electromagnetic ion cyclotron waves, J. Geophys. Res., 112, A04207, doi:10.1029/ 2006JA011993.

Trakhtengerts, V. Y. (1995), Magnetosphere cyclotron maser: Backward wave oscillator generation regime, J. Geophys. Res., 100(A9), 17,205-17,210, doi:10.1029/95JA00843.

Trakhtengerts, V. Y. (1999), A generation mechanism for chorus emission, Ann. Geophys., 17, 95-100.

Tsurutani, B. T. and E. J. Smith (1974), Postmidnight chorus: a substorm phenomenon, J. Geophys. Res., 79(1), 118-127, doi:10.1029/ JA079i001p00118.

Xiao, F., R. M. Thorne, and D. Summers (1998), Instability of electromagnetic R-mode waves in a relativistic plasma, Phys. Plasmas, 5, 7, 2489-2497, doi:10.1063/1.872932.

Y. Katoh, Department of Geophysics, Graduate School of Science, Tohoku University, Sendai, Miyagi 980-8578, Japan. (yuto@stpp.gp tohoku.ac.jp)

Y. Omura, Research Institute for Sustainable Humanosphere, Kyoto University, Uji, Kyoto 611-0011, Japan. (omura@rish.kyoto-u.ac.jp) 